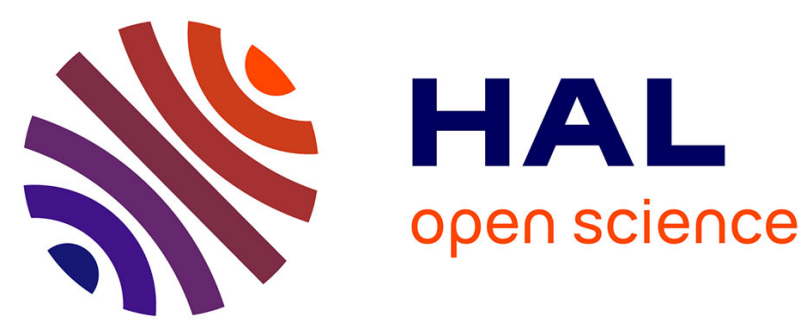

\title{
Geometric Multi-Wavelet Total Variation for SAR Image Time Series Analysis
}

Abdourrahmane Atto, Anoumou Kemavo, Jean-Paul Rudant, Grégoire

Mercier

\section{To cite this version:}

Abdourrahmane Atto, Anoumou Kemavo, Jean-Paul Rudant, Grégoire Mercier. Geometric MultiWavelet Total Variation for SAR Image Time Series Analysis. International Conference on Information Fusion (FUSION 2018), Jul 2018, Ccambridge, United Kingdom. hal-01794292

\section{HAL Id: hal-01794292 \\ https://hal.science/hal-01794292}

Submitted on 17 May 2018

HAL is a multi-disciplinary open access archive for the deposit and dissemination of scientific research documents, whether they are published or not. The documents may come from teaching and research institutions in France or abroad, or from public or private research centers.
L'archive ouverte pluridisciplinaire HAL, est destinée au dépôt et à la diffusion de documents scientifiques de niveau recherche, publiés ou non, émanant des établissements d'enseignement et de recherche français ou étrangers, des laboratoires publics ou privés. 


\section{Geometric Multi-Wavelet Total Variation for SAR Image Time Series Analysis}

\author{
Abdourrahmane M. ATTO \\ Université Savoie Mont Blanc \\ LISTIC, Annecy, France \\ abdourrahmane.atto@univ-smb.fr \\ Grégoire MERCIER \\ eXo maKina \\ Digital Technologies, Paris, France \\ gregoire.mercier@exomakina.fr
}

\author{
Anoumou Kemavo \\ ONF International \\ Nogent-sur-Marne, France \\ anoumou.kemavo@onfinternational.com
}

\author{
Jean-Paul RUDANT \\ Université Paris-Est Marne-la-Vallée \\ LaSTIG/IGN, MATIS, Paris, France \\ jean-paul.rudant@u-pem.fr
}

\begin{abstract}
A time series issued from modern synthetic aperture radar satellite imaging sensors is a huge dataset composed by many hundreds of million pixels when observing large-scale earth structures such as big forests or glaciers. A concise monitoring of these large scale structures for anomaly spotting thus requires loading and analyzing huge spatio/polarimetric multi-temporal image series. The contributions of the present paper for the sake of parsimonious analysis of such huge datasets are associated with a framework having two main processing stages. The first stage is the derivation of an index called geometric multi-wavelet total variation for fast and robust anomaly spotting. This index is useful for identifying significant abnormal patterns appearing as geo-spatial non-stationarities in multi-wavelet total variation map. The second stage consists in the proposal of a concise asymmetric multi-date change information matrix on regions associated with significant multi-wavelet total variations. This stage is necessary for a fine characterization of change impacts on existing geo-spatial structures. Experimental tests based on Sentinel-1 data show relevant results on a wide Amazonian forest surrounding the Franco-Brazilian Oyapock Bridge.
\end{abstract}

Index Terms-Multi-Wavelets ; Information fusion ; Geometric differencing ; Multivariate Synthetic Aperture Radar; Image Time Series.

\section{INTRODUCTION}

$\mathbf{I}$ NFORMATION retrieval from long-time series of huge spatial Synthetic Aperture Radar (SAR) images associated with several polarization modalities requires identifying statistics and similarity measures that can lead to fast and robust computations over billions of pixels. Among the standard statistics/measures adapted to this issue, ratio operators are the most straightforward change indicators and are known to be relevant on intensities or amplitudes of SAR multiplicative scatterings.

When the SAR sensor resolution is not high enough ${ }^{1}$, recent literature has investigated some refinements of log-ratio change measure by:

The work was supported by PHOENIX ANR-15-CE23-0012 grant of the French National Agency of Research.

${ }^{1}$ This is the case for the recent Sentinel imaging constellation, among other on-board satellite SAR sensors.
- filtering data before applying log-ratio measure [1] ;

- filtering log-ratio measures (by using mean operators in [2], [3] or by using wavelet transforms in [4], the issue here is obtaining a finer wavelet based approximation ${ }^{2}$ ) ;

- log-ratio over non-necessarily connected pixels by means of graph theory [5] or spectrally invariant keypoints [6].

Other results such as [7], [8] concern the analysis of log-ratio statistical properties in order to ease selection of detection threshold, segmentation or classification.

Despite its relevancy, the ratio measure can fail in detecting changes when several errors affect data at different levels (diffusion of scatterers, calibration and registration errors, etc.) whereas filtering the data (prior to computing the ratio) can blur change information, especially for the coarse resolution offered by public-level satellite data.

For deriving a robust, generalized ratio-like measure with as straightforward implementation as the standard ratio operator, one can first remark that the latter is a particular case of geometric wavelet operators [9]. he second remark is that different of such geometric wavelet filters can lead to alternative ratio operators that can balance the possible mis-detections of the standard ratio operator, in addition to offering multi-scale ratioing.

The first issue addressed in this paper is then fusing multiple evaluations from several classes of geometric wavelet filters, including the one corresponding to the standard ratio operator, in order to obtain robustness of the measure while keeping a fast and easy implementation. The index proposed from such several geometric wavelet operators for the sake of pixel-level anomaly spotting will be the aggregation (Total Variation, $\mathrm{TV}$ ) of mono-wavelet measures and will be called multiwavelet TV. This index stands out from standard wavelet transform based fusion techniques [10], [11], [12] in the sense that it applies only on difference/ratio measures (temporal

\footnotetext{
${ }^{2} \overline{\text { Note that }}$ this approximation is a regularized version where the scaling function of the standard wavelet transform plays a major role.
} 
trends/approximations are not considered as change information).

From this index, pixels having abnormal dynamics will be associated with large multi-wavelet TVs. In addition, a recursive regularization framework addressed in the paper allows for spotting the surrounding abnormal change area. This recursive regularization is adapted from [13] in order to take multivariate images into account.

Finally, when pixels with large multi-wavelet TV are located in a dynamic neighborhood, we propose a focus on the surrounding area with the sake of multiple change detection and evolution monitoring. Since the surrounding area is expected to be small in practice (not as huge as the entire image), then a relevant feature for region based texture similarity evaluation is provided in terms of Multi-Date Divergence Matrices (MDDM) introduced in [14]. The MDDM will be computed with respect to divergence information upon probabilistic features attached to a multiscale wavelet representation of the area with significant multi-wavelet TV. In contrast with [14], the MDDM proposed in this paper will be asymmetric to encompass dual-polarimetric dissimilarities in the information matrix.

The paper is organized as follows. Section II proposes the geometric multi-wavelet total variation dynamicity measure and addresses the identification of dynamic pixels and their surrounding areas in time series of images. Section III proposes, for the surrounding areas affected by high dynamicity TV measures, asymmetric MDDMs computed from geometric wavelet transforms for highlighting abrupt and progressive changes, as well as multiple changes in the series of images. Section IV addresses experimental results on a FrancoBrazilian test site which is part of the Amazonian forest. Section V concludes the work. A summary of the framework proposed in the paper for handling long image time series of huge spatial sizes is given by Figure 1.

\section{Stage 1: Identification of Areas with LARge WAVELET TOTAL VARIATION}

\section{A. Notation}

In what follows, $\mathcal{I}=\left\{\mathcal{I}_{k}\right\}_{k=1, \ldots, M}$ is a Polarimetric SAR (PolSAR) Image Time Series (ITS) composed by $M$ coregistered images of the same scene and such that $\mathcal{I}_{k}=\mathcal{I}\left[t_{k}\right]$, where $k / t_{k}$ refers to the acquisition date. The pixel $\mathcal{I}_{k}^{\mathcal{P}}(x, y)$ is with spatial location $(x, y)$, sampling date $k$ and polarization information $\mathcal{P}$. Polarimetry information $\mathcal{P}$ can be provided in terms of one among the following features:

- Scattering matrix $(\mathcal{P} \triangleq \mathcal{S}$, where symbol $\triangleq$ stands for notation convention): we have $\mathcal{I}_{k}^{\mathcal{S}}(x, y)=\mathcal{S}\left[t_{k}\right](x, y) \triangleq \mathcal{S}$ where

$$
\mathcal{S}=\left(\begin{array}{cc}
S_{1,1} & S_{1,2} \\
S_{2,1} & S_{2,2}
\end{array}\right)
$$

when omitting variables $t_{k},(x, y)$ and by assuming that the scattering information is associated with quad polarimetry SAR acquisition modalities (in this case,
$S_{p, q}$ is the SAR response with respect to signal transmission/receive operating at polarization configurations ${ }^{3}$ $p / q)$.

- Covariance matrix $(\mathcal{P} \triangleq \mathcal{C})$ : $\mathcal{I}_{k}^{\mathcal{C}}(x, y)=\mathcal{C}\left[t_{k}\right](x, y) \triangleq \mathcal{C}$ with:

$\mathcal{C}=$

$$
\left(\begin{array}{ccc}
\left|S_{1,1}\right|^{2} & \frac{S_{1,1}\left(S_{1,2}+S_{2,1}\right)^{*}}{\sqrt{2}} & S_{1,1} S_{2,2}^{*} \\
\frac{\left(S_{1,2}+S_{2,1}\right) S_{1,1}^{*}}{\sqrt{2}} & \frac{\left|S_{1,2}+S_{2,1}\right|^{2}}{2} & \frac{\left(S_{1,2}+S_{2,1}\right) S_{2,2}^{*}}{\sqrt{2}} \\
S_{2,2} S_{1,1}^{*} & \frac{S_{2,2}\left(S_{1,2}+S_{2,1}\right)^{*}}{\sqrt{2}} & \left|S_{2,2}\right|^{2}
\end{array}\right)
$$

This matrix is obtained from equation $\mathcal{C}=\underline{\mathbf{k}}_{3 \mathrm{~L}} \overline{\mathbf{k}_{3 \mathrm{~L}}^{*}}$ where

$$
\underline{\mathbf{k}}_{3 \mathrm{~L}}=\left(\begin{array}{c}
S_{1,1} \\
\left(S_{1,2}+S_{2,1}\right) / \sqrt{2} \\
S_{2,2}
\end{array}\right)
$$

is the lexicographic vector associated with scattering matrix $\mathcal{S}$

- Coherency matrix $(\mathcal{P} \triangleq \mathcal{T}): \mathcal{I}_{k}^{\mathcal{T}}(x, y)=\mathcal{T}\left[t_{k}\right](x, y) \triangleq \mathcal{T}$ with:

$$
\mathcal{T}=\underline{\mathbf{k}}_{3 \mathrm{P}} \underline{\mathbf{k}}_{3 \mathrm{P}}^{*}
$$

where $\underline{\mathbf{k}}_{3 \mathrm{P}}$ is the Pauli vector defined by:

$$
\underline{\mathbf{k}}_{3 \mathrm{P}}=\frac{1}{\sqrt{2}}\left(\begin{array}{c}
S_{1,1}+S_{2,2} \\
S_{1,1}-S_{2,2} \\
S_{1,2}+S_{2,1}
\end{array}\right)
$$

Expansion of $\mathcal{T}$ with respect to scattering coefficients is omitted due to its large size. But it is worth noting that coefficients of $\mathcal{T}$ are associated to the so-called Huynen parameters $\mathbf{A}_{0}, \mathbf{B}_{0}, \mathbf{B}_{1}, \mathbf{C}, \mathbf{D}, \mathbf{E}, \mathbf{F}, \mathbf{G}, \mathbf{H}$ through the relation:

$$
\mathcal{T} \triangleq \underline{\mathbf{k}}_{3 \mathrm{P}} \overline{\underline{\mathbf{k}}_{3 \mathrm{P}}^{*}}=\left(\begin{array}{ccc}
2 \mathbf{A}_{0} & \mathbf{C}-i \mathbf{D} & \mathbf{H}+i \mathbf{G} \\
\mathbf{C}+i \mathbf{D} & \mathbf{B}_{0}+\mathbf{B}_{1} & \mathbf{E}+i \mathbf{F} \\
\mathbf{H}-i \mathbf{G} & \mathbf{E}-i \mathbf{F} & \mathbf{B}_{0}-\mathbf{B}_{1}
\end{array}\right)
$$

When only dual-polarimetry information are available, then we consider a 3-variate dual-PolSAR $\mathrm{RGB}^{4}$ vector $\mathcal{I}_{k}^{\mathcal{V}}(x, y)=\mathcal{V}\left[t_{k}\right](x, y) \triangleq \mathcal{V}$ in terms of the following scattering compositions (case $\mathrm{SLC}^{5}$ ), respectively covariance magnitude compositions (case $\mathrm{GRD}^{6}$ ):

$$
\begin{gathered}
\mathcal{V}^{\mathrm{SLC}}=\left(\begin{array}{c}
S_{2,2} \\
S_{2,1} \\
S_{2,2} / S_{2,1}
\end{array}\right) \| \mathcal{V}^{\mathrm{SLC}}=\left(\begin{array}{c}
S_{1,1} \\
S_{1,2} \\
S_{1,1} / S_{1,2}
\end{array}\right) \\
\mathcal{V}^{\mathrm{GDR}}=\left(\begin{array}{c}
\left|S_{2,2}\right|^{2} \\
\left|S_{2,1}\right|^{2} \\
\left|S_{2,2} / S_{2,1}\right|^{2}
\end{array}\right) \| \mathcal{V}^{\mathrm{GDR}}=\left(\begin{array}{c}
\left|S_{1,1}\right|^{2} \\
\left|S_{1,2}\right|^{2} \\
\left|S_{1,1} / S_{1,2}\right|^{2}
\end{array}\right)
\end{gathered}
$$

depending on the pair available among scattering coefficients $\left(S_{2,2}, S_{2,1}\right)$ and $\left(S_{1,1}, S_{1,2}\right)$, where symbol \| refers to exclusive "or" operator. Notation $\mathcal{P} \triangleq \mathcal{V}$ applies for the latter acquisition specificities.

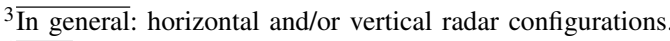

${ }^{4} \overline{\mathrm{RGB}}$ : color model associated with Red, Green and Blue.

${ }^{5} \overline{\text { SLC }}$ : Single Look Complex valued SAR products.

${ }^{6} \overline{\mathrm{GRD}}$ : Ground Range Detected, real valued post-processed SAR products. 


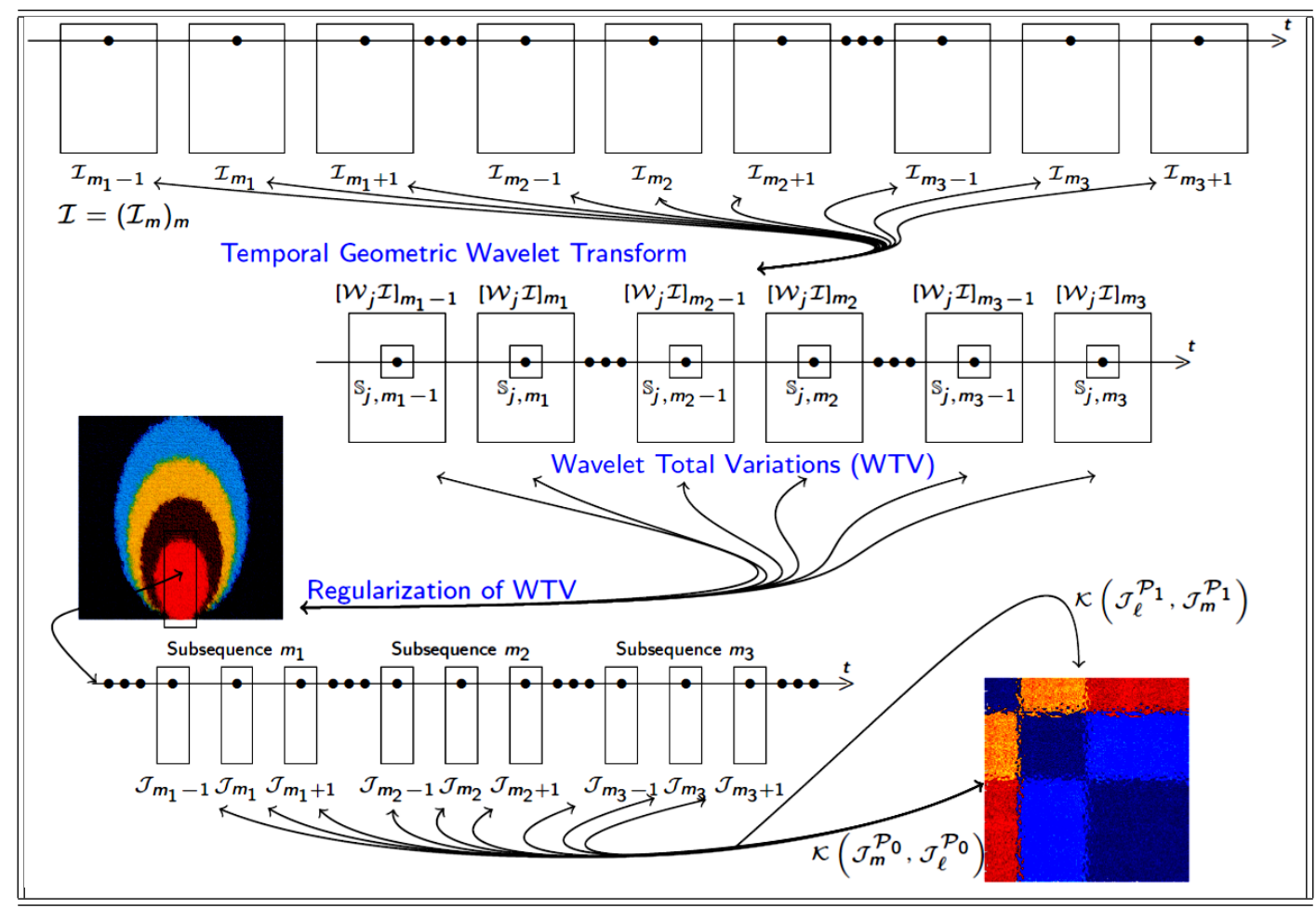

Fig. 1. Diagram of ITS analysis. WTV are computed at pixel levels over huge spatial areas. Regions with large WTV are then analyzed by MDDMs (sub-image/patch levels).

\section{B. Geometric Wavelet Total Variation}

Assume that spatial variables $(x, y)$ cover a huge area. We need a fast and efficient pre-processing that makes the identification of areas affected by significant events/changes/evolution possible. In particular, both abrupt and slow range progressive changes must result in high alarm responses for a relevant pre-processing.

A convenient solution is the temporal Wavelet Total Variation (WTV) index defined here as the sum of magnitudes of wavelet ${ }^{7}$ coefficients when the wavelet transform apply with respect to the time axis.

Moreover, since using different wavelets can lead to a richer framework for capturing a wide class of non-stationarities, we consider from now on, a set of $J$ temporal causal ${ }^{8}$ geometric wavelet transforms $\left(\mathcal{W}_{j}\right)_{1 \leqslant j \leqslant J}$ that deliver a set of PolSAR variability features where, for any given $\mathcal{P}$, the spatial sequence of coefficients at date $k$ is obtained for every $(x, y)$ by the following multiplicative fusion operator:

$$
\left[\mathcal{W}_{j} \mathcal{I}\right]^{\mathcal{P}}[k](x, y)=\prod_{\ell=0}^{L_{j}-1}\left(\mathcal{I}_{k-\ell}^{\mathcal{P}}(x, y)\right)^{\mathcal{W}_{j}[\ell]}
$$

In this equation, causal implementation means that we have considered shifted versions of the wavelet impulse response so that $\mathcal{W}$ has effective support: $\left\{0,1,2, \ldots, L_{j}-1\right\}$.

\footnotetext{
${ }^{7}$ A wavelet function is assumed to admit at least one vanishing moment in order to be considered as a generalized differential operator.

${ }^{8} \overline{\text { Causality }}$ is imposed here for operational purpose and easy-to-update analysis schemes.
}

For instance, we will use the following wavelet transforms, where variables $\mathcal{P}$ and $(x, y)$ are omitted for the sake of readability:

- Level-1 Haar (notation Haar-1 hereafter) geometric wavelet transform of $\mathcal{I}$ :

$$
\left[\mathcal{W}_{1} \mathcal{I}\right][k]=\left[\mathcal{W}_{\text {Haar- } 1} \mathcal{I}\right][k]=\mathcal{I}_{k}^{\frac{1}{2}} \mathcal{I}_{k-1}^{-\frac{1}{2}}
$$

- Biorthogonal (notation $\mathrm{Bi}-\perp$ hereafter) geometric wavelet transform of $\mathcal{I}$ :

$$
\left[\mathcal{W}_{2} \mathcal{I}\right][k]=\left[\mathcal{W}_{\mathrm{Bi} \perp} \mathcal{I}\right][k]=\mathcal{I}_{k}^{\frac{1}{3}} \mathcal{I}_{k-1}^{-\frac{2}{3}} \mathcal{I}_{k-2}^{\frac{1}{3}}
$$

- Level-2 Haar (notation Haar-2 hereafter) geometric wavelet transform of $\mathcal{I}$ :

$$
\left[\mathcal{W}_{3} \mathcal{I}\right][k]=\left[\mathcal{W}_{\text {Haar }-2} \mathcal{I}\right][k]=\mathcal{I}_{k}^{\frac{1}{4}} \mathcal{I}_{k-1}^{\frac{1}{4}} \mathcal{I}_{k-2}^{-\frac{1}{4}} \mathcal{I}_{k-3}^{-\frac{1}{4}}
$$

In these equations, $\sum_{\ell} \mathcal{W}_{j}[\ell]=0$ to ensure vanishing moment condition. As a consequence, any $\left[\mathcal{W}_{j} \mathcal{I}\right]$ is a geometric differencing (ratioing) operator. Quantity $\left[\mathcal{W}_{j} \mathcal{I}\right][k]$ is thus an indicator of the amount/number of geometric changes obtained at date $k$ and computed with respect to the $L_{j}-1$ foregoing dates, where $L_{j}$ is the length of the wavelet support.

Remark 1 (Terminology): We will say that system:

- $\{$ Haar-1, Haar-2\} is mono-wavelet but multi-scale (scales $2^{1}$ for Haar-1 and $2^{2}$ for Haar-2) ;

- $\{$ Haar-1, Bi- $\perp\}$ is multi-wavelet but mono-scale (scale $2^{1}$ for both Haar-1 and Bi- $\perp$ wavelets) ;

- $\{$ Haar-1, Bi- $\perp$, Haar-2 $\}$ is multi-scale multi-wavelet. 
We will consider either the multi-scale multi-wavelet system $\{$ Haar-1, Bi- $\perp$, Haar- 2$\}$ or the mono-wavelet multi-scale system $\{$ Haar-1, Haar-2, Haar-3\} where Haar-3 is derived similarly with respect to Haar-1 and Haar-2.

Let us define the Geometric WTV ${ }^{9}$ (GWTV) observed in the $M$-length time series $\mathcal{I}$ by using $\mathcal{W}_{j}$ as:

$$
\Theta_{j, M}=\sum_{k=L_{j}}^{M}|\log |\left[\mathcal{W}_{j} \mathcal{I}\right][k]||
$$

The use of $\log$ in GWTV given by Eq. (5) is required mainly for the sake of displaying the multiplicative increments into additive scales. The absolute value operating on the log allows to keep all magnitudes detected over time: without this absolute value, positive and negative change responses over time can annihilate in the sum, resulting in an inaccurate "stability" decision.

Remark 2 (GWTV of complex valued features): Transform $\mathcal{W}_{j} \mathcal{I}$ of Eq. (1) involves:

- only real numbers when $\mathcal{I}=\mathcal{V}^{\mathrm{GDR}}$ or

- complex and real numbers when $\mathcal{I}=\mathcal{S} / \mathcal{C} / \mathcal{T} / \mathcal{V}^{\text {SLC }}$.

In the latter case, $\mathcal{W}_{j}$ implementation involves ratios of PolSAR moduli weighted by complex exponentials (associated with wavelet based differences of phase information). However, Eq. (5) remains real, non-negative, because it applies on the moduli of the complex wavelet coefficients obtained by using Eq. (1).

Remark 3 (Updating GWTV): For huge data processing in operational multi-temporal context, it is important to be able to update GWTV of Eq. (5) without significant computational load when a new observation is available. Note that the updating process only requires:

- retrieval of image $\mathcal{I}_{M+1}$;

- computation of the wavelet based image coefficients $\left[\mathcal{W}_{j} \mathcal{I}\right][M+1]$ by using one among Eqs. (2), (3), (4) or both of these equations, this computation involves loading only the $L_{j}-1$ foregoing images (for instance loading $\left\{\mathcal{I}_{M}\right\},\left\{\mathcal{I}_{M}, \mathcal{I}_{M-1}\right\}$ or $\left\{\mathcal{I}_{M}, \mathcal{I}_{M-1}, \mathcal{I}_{M-2}\right\}$ respectively when considering Haar-1, Bi- $\perp$ or Haar-2 wavelets);

- computation of

$$
\Theta_{j, M+1}=\Theta_{j, M}+|\log |\left[\mathcal{W}_{j} \mathcal{I}\right][M+1]||
$$

Thus, the overall update processing is with low data-load and small computational-complexity.

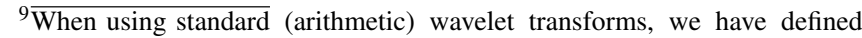
(see the beginning of section II-B), the WTV as:

$$
\Theta_{j, M}=\sum_{k=L_{j}}^{M}\left|\left[\mathcal{W}_{j} \mathcal{I}\right][k]\right|
$$

In practice, only the so-called "detail" wavelet coefficients have to be taken into account in the above sum: when using a standard wavelet decomposition, it is necessary to discard approximation coefficients from this sum because they are associated with a scaling (not a wavelet) function.
Finally, with the sake of taking advantage of diversities induced by the use of different wavelet shapes, we define the Geometric Multi-Wavelet ${ }^{10}$ WTV (GMWTV) for the $M$ length ITS $\mathcal{I}$ as the following additive fusion of geometric mono-wavelet integrals:

$$
\Theta_{M}=\sum_{j} \alpha_{j} \Theta_{j, M}
$$

where $j$ ranges over a set of wavelet indices (or scale parameters) and sequence $\left(\alpha_{j}\right)_{j}$, satisfying $\sum_{j} \alpha_{j}=1$, is a sequence of weights associated with wavelet relevancy for discriminating a given type of change/evolution.

\section{Regularization of WTV}

Despite the doubly additive and multiplicative fusions involved in GMWTV, the rationale is to admit that anomaly/evolution information contained in any GWTV (thus GMWTV) can be slightly affected by errors issued from upstream SAR pre-processing steps (imperfect calibration, inconsistent geometric projectors in case of terrain correction and co-registration errors). To enforce regularity in presence of spatial dynamics, the following proposes regularization of WTV as a spatial/polarimetric processing of temporal change information. Given a WTV $\Theta_{j, M}$, this regularization, inspired by [13], applies from the following algorithm:

- Step 1: $\Theta_{j, M}^{\triangleleft p} \leftarrow \Theta_{j, M}$, where $\leftarrow$ denotes the assignment symbol.

- Step 2: Compute the continuous Hilbert-Peano space filling curve associated with spatial image indices: the outputs are two reshaped vectors $U$ and $V$ and the univoque transform:

$$
\Theta_{j, M}^{\triangleleft p}(U(z), V(z))=\Theta_{j, M}^{\triangleleft p \vee}(x, y)
$$

which provides the one-dimensional path (single variable $z$ ) making a continuous move through the image spatial coordinates possible.

- Step 3: For $z$ ranging from 1 to the end of the 2D HilbertPeano path, extract the set ${ }^{11}$ :

$$
\begin{aligned}
\mathbb{S}_{j, M, z}[ & \left.\eta_{1}, \eta_{2}\right]= \\
\Theta_{j, M}^{\triangleleft} \boldsymbol{\nabla} & \left(U(z)-c-1+\eta_{1}, V(z)-c-1+\eta_{2}\right)
\end{aligned}
$$

for $1 \leqslant \eta_{1}, \eta_{2} \leqslant 2 c+1$, then overwrite the value $\Theta_{j, M}^{\triangleleft p}(U(z), V(z))$ as follows:

$$
\Theta_{j, M}^{\triangleleft p}(U(z), V(z)) \leftarrow \zeta
$$

where

$$
\zeta=\arg \min _{w \in \mathbb{S}_{j, M, z}} \sum_{\eta_{1}, \eta_{2}=1}^{2 c+1}\left\|\mathbb{S}_{j, M, z}\left[\eta_{1}, \eta_{2}\right]-w\right\|_{\ell^{p}}^{p}
$$

${ }^{10} \overline{\text { Same terminology }}$ for either or both multi-scale/multi-wavelet since multi-scale can be seen as a particular case of multi-wavelet.

${ }^{11} \overline{\text { Depending on }}$ the data type in $\mathcal{P}$, the set $\mathbb{S}_{j, M, z}$ may be composed by scalars, vectors or matrices. 
before computing $\mathbb{S}_{j, M, z+1}$, followed by the calculus of $\Theta_{j, M}^{\triangleleft p}(U(z+1), V(z+1)), \ldots$, loop until the end of the Hilbert-Peano path.

This regularization, with efficiency proven in [13] for scalar images among which SAR intensity data, allows for penalizing spatial neighbors with small $\ell^{p}$ norm while enhancing pixels associated with large change responses. When $p=1$, this corresponds to a recursive median computation that purges outliers with a segmentation-like effect.

Remark 4 (Joint versus independent spatio/polarimetric regularization): In Eq. (8), the $\ell^{p}$ norm can apply:

- to spatial variable only, for any given polarimetric information, that is: $\mathbb{S}_{j, M, z}$ is a series of scalar data (reshaped spatial neighborhood),

- to both spatio-polarimetric variables: $\mathbb{S}_{j, M, z}$ is a series of multivariate PolSAR data associated with the given spatial neighborhood and vector/matrix $\ell^{p}$ norms are under consideration.

In the former case, regularization concerns polarimetry channels independently whereas in the latter case, all available polarimetry information are integrated in the $\ell^{p}$ norm. In practice, from several tests on data associated with different sensors, we can conclude that: for systems delivering lower $\left(S_{1,2}, S_{2,1}\right)$ scattering responses than $\left(S_{1,1}, S_{2,2}\right)$, the marginal (channel independent processing) is more relevant. Otherwise, the joint spatio-polarimetric neighborhood leads to a more PolSAR discriminant regularization.

Remark 5 (Regularization before of after multi-wavelet fusion): Alternatively, rather than regularizing the GWTV given by Eq. (5) for any $j$, one may consider regularizing the fused GMWTV (defined by Eq. (6)) directly. In practice, as long as the number of wavelets is larger than 2, we observe that it is more convenient to consider the latter for obtaining a good compromise in terms of computational complexity and performance.

\section{Stage 2: Multi-Date Information Retrieval FOR AREAS WITH LARGE GMWTV}

The analysis proposed in this section concerns an area or a region with large GMWTV. We keep the same notation $\mathcal{I}$ for the image/region/area/subimage/patch whatever the spatial size of interest. MDDMs proposed in [14] for mono-channel SAR images are tools for visualizing several categories of changes that can affect an ITS. The MDDM is composed by change information between pairwise observations $\mathcal{I}_{m}$ and $\mathcal{I}_{\ell}$ for all available couples of dates $(m, \ell)$. It corresponds to the matrix:

$$
\mathbf{K}=\left(\mathcal{K}\left(\mathcal{I}_{m}, \mathcal{I}_{\ell}\right)\right)_{1 \leqslant m, \ell \leqslant M}
$$

where $\mathcal{K}$ is an appropriate dissimilarity measure, for instance the symmetric Kullback-Leibler divergence:

$$
\mathcal{K}\left(X_{1}, X_{2}\right)=\mathcal{K}\left(X_{1} \| X_{2}\right)+\mathcal{K}\left(X_{2} \| X_{1}\right)
$$

where $\mathcal{K}\left(X_{1} \| X_{2}\right)$ is given by:

$$
\mathcal{K}\left(X_{i} \| X_{j}\right)=\int_{\mathbb{R}} f_{X_{i}}(x) \log \frac{f_{X_{i}}(x)}{f_{X_{j}}(x)} d x, \quad i, j=1,2
$$

with $f_{X_{1}}$ and $f_{X_{2}}$ being the PDFs (Probability Distribution Functions) of random variables $X_{1}$ and $X_{2}$ respectively.

\section{A. Case of dual-PolSAR ITS}

For dual PolSAR data, we consider the first two components of the feature vector $\mathcal{V}^{\text {GRD }}$ (or $\mathcal{V}^{\text {SLC }}$ ) introduced in Section II-A. The PolSAR variables can be noted $\mathcal{P}=\left(\mathcal{P}_{\epsilon}, \mathcal{P}_{1-\epsilon}\right)$, for $\epsilon \in\{0,1\}$, with: $\left(\mathcal{P}_{\epsilon}, \mathcal{P}_{1-\epsilon}\right)=(\mathrm{VV}, \mathrm{VH})$ or $(\mathrm{HH}, \mathrm{HV})$ for Sentinel-1 data, where $\mathrm{H} / \mathrm{V}$ refers to Horizontal/Vertical polarizations.

We define the dual-PolSAR MDDM as:

$$
\begin{aligned}
& \mathbf{K}_{\mathcal{P}}= \\
& \quad\left(\mathcal{K}\left(\mathcal{I}_{m}^{\mathcal{P}_{\epsilon}}, \mathcal{I}_{\ell}^{\mathcal{P}_{\epsilon}}\right) \mathbb{1}_{\{m \leqslant \ell\}}+\mathcal{K}\left(\mathcal{I}_{m}^{\mathcal{P}_{1-\epsilon}}, \mathcal{I}_{\ell}^{\mathcal{P}_{1-\epsilon}}\right) \mathbb{1}_{\{m \geqslant \ell\}}\right)_{1 \leqslant m, \ell \leqslant M}
\end{aligned}
$$

From this definition and when $\left(\mathcal{P}_{\epsilon}, \mathcal{P}_{1-\epsilon}\right)=(\mathrm{VV}, \mathrm{VH})$, then the upper triangular divergence matrix represents $\mathrm{VV}$-based dissimilarity measurements and the lower triangular divergence matrix provides $\mathrm{VH}$-based dissimilarity measurements.

As a consequence, the corresponding MDDM is a nonnecessarily symmetric change information matrix highlighting both VV and VH PolSAR dissimilarities over time: depending on the ground scatterer imaged, changes may be observable either on the lower (respectively upper) matrix components or on both parts of this matrix. The goal is to clearly emphasize a discriminant dual-PolSAR change information.

For any given $\epsilon \in\{0,1\}$, the $\mathcal{K}\left(\mathcal{I}_{m}^{\mathcal{P}_{\epsilon}}, \mathcal{I}_{\ell}^{\mathcal{P}_{\epsilon}}\right)$ dissimilarity will be computed upon the coefficients of a spatial ${ }^{12}$ standard Stationary Wavelet Transform (SWT) W for evaluating between-date texture features:

$$
\begin{aligned}
\mathcal{K}\left(\mathcal{I}_{m}^{\mathcal{P}_{\epsilon}}, \mathcal{I}_{\ell}^{\mathcal{P}_{\epsilon}}\right) \triangleq & \mathcal{K}\left(\mathbf{W}_{J, 0}^{\Re}\left[\mathcal{I}_{m}^{\mathcal{P}_{\epsilon}}\right], \mathbf{W}_{J, 0}^{\Re}\left[\mathcal{I}_{\ell}^{\mathcal{P}_{\epsilon}}\right]\right) \\
+ & \mathcal{K}\left(\mathbf{W}_{J, 0}^{\Im}\left[\mathcal{I}_{m}^{\mathcal{P}_{\epsilon}}\right], \mathbf{W}_{J, 0}^{\Im}\left[\mathcal{I}_{\ell}^{\mathcal{P}_{\epsilon}}\right]\right) \\
+ & \sum_{\substack{j \in\{1,2 \ldots, J\} \\
n \in\{1,2,3\}}} \mathcal{K}\left(\left|\mathbf{W}_{j, n}\left[\mathcal{I}_{m}^{\mathcal{P}_{\epsilon}}\right]\right|,\left|\mathbf{W}_{j, n}\left[\mathcal{I}_{\ell}^{\mathcal{P}_{\epsilon}}\right]\right|\right)
\end{aligned}
$$

where $\mathbf{W}_{j, n}\left[\mathcal{I}_{m}^{\mathcal{P}_{\epsilon}}\right], \mathbf{W}_{j, n}\left[\mathcal{I}_{\ell}^{\mathcal{P}_{\epsilon}}\right]$ are the level $(j, n)$ SWT coefficients of $\mathcal{I}_{m}^{\mathcal{P}_{\epsilon}}, \mathcal{I}_{\ell}^{\mathcal{P}_{\epsilon}}$ respectively and $\Re / \Im$ are real/imaginary parts of a complex number. The direct sum used in Eq. (11) assumes that for any given date $m$, the variables $\left(\mathbf{W}_{j, n}\left[\mathcal{I}_{m}^{\mathcal{P}_{\epsilon}}\right]\right)_{j, n}$ are approximately independent. This is a property of wavelet transforms that is known to depend on: $(i)$ the input random process and $(\mathrm{ii})$ the wavelet filter properties.

\section{B. Case of quad-PolSAR ITS}

For quad-PolSAR time series, we are concerned by one of the matrix features $\mathcal{S}, \mathcal{C}, \mathcal{T}$ described in Section II-A. The number of variables being large, we can distinguish two issues: either using a discriminant analysis per polarimetry channel (which leads to one MDDM per channel), or deriving a single MDDM. The latter is more parsimonious for fine quadPolSAR $^{13}$ since the scatterer responses are expected to be

\footnotetext{
$12 \overline{\text { Texture-level }}$ analysis, in contrast with the first stage analysis operating at pixel-level.

${ }^{13} \overline{\text { RADARSAT-2 satellite }}$ offers such possibility, other sources being airborne SAR imaging systems.
} 
significant in any PolSAR channel. In this case, we define the MDDM from fused divergences:

$$
\mathcal{K}\left(\mathcal{I}_{m}^{\mathcal{P}}, \mathcal{I}_{\ell}^{\mathcal{P}}\right)=\sum_{p} \sum_{q} \mathcal{K}\left(\mathcal{I}_{m}^{\mathcal{P}_{p, q}}, \mathcal{I}_{\ell}^{\mathcal{P}_{p, q}}\right)
$$

where

$$
\begin{aligned}
\mathcal{K}\left(\mathcal{I}_{m}^{\mathcal{P}_{p, q}}, \mathcal{I}_{\ell}^{\mathcal{P}_{p, q}}\right) \triangleq \mathcal{K}\left(\mathbf{W}_{J, 0}^{\Re}\left[\mathcal{I}_{m}^{\mathcal{P}_{p, q}}\right], \mathbf{W}_{J, 0}^{\Re}\left[\mathcal{I}_{\ell}^{\mathcal{P}_{p, q}}\right]\right) \\
+\mathcal{K}^{\mathcal{K}}\left(\mathbf{W}_{J, 0}^{\Im}\left[\mathcal{I}_{m}^{\mathcal{P}_{p, q}}\right], \mathbf{W}_{J, 0}^{\Im}\left[\mathcal{I}_{\ell}^{\mathcal{P}_{p, q}}\right]\right) \\
+\sum_{\substack{j \in\{1,2 \ldots, J\} \\
n \in\{1,2,3\}}} \mathcal{K}\left(\left|\mathbf{W}_{j, n}\left[\mathcal{I}_{m}^{\mathcal{P}_{p, q}}\right]\right|,\left|\mathbf{W}_{j, n}\left[\mathcal{I}_{\ell}^{\mathcal{P}_{p, q}}\right]\right|\right)
\end{aligned}
$$

As in Section III-A above, the sum in Eq. (12) assumes that wavelet contributions are approximately independent. Otherwise, a weighted sum can be investigated.

To conclude this section, it is worth mentioning that the divergence expressed by Eq. (10) requires either a parametric PDF model or a non-parametric estimate of the PDF. Hereafter, we take again advantage of the regularization operated by wavelets on random processes for associating, with a preliminary validation stage:

- Generalized Gaussian (GG) models to real/imaginary parts of approximation coefficients and

- Weibull (WBL) models to magnitudes of wavelet details. Therefore, on the one hand, we will associate to PolSAR spatial approximation coefficients, the scale $\alpha$ and shape $\beta$ of a GG distribution: if $X_{1}=\mathbf{W}_{J, 0}^{\Re / \Im}\left[\mathcal{I}_{m}^{\mathcal{P}_{\epsilon}}\right]$ and $X_{2}=\mathbf{W}_{J, 0}^{\Re / \Im}\left[\mathcal{I}_{\ell}^{\mathcal{P}_{\epsilon}}\right]$ are the corresponding GG variables, then the symmetric divergence of Eq. (9) has the following form [15]:

$$
\begin{aligned}
\mathcal{K}\left(X_{1}, X_{2}\right)= & \left(\frac{\alpha_{1}}{\alpha_{2}}\right)^{\beta_{2}} \frac{\Gamma\left(\frac{1+\beta_{2}}{\beta_{1}}\right)}{\Gamma\left(1 / \beta_{1}\right)} \\
& +\left(\frac{\alpha_{2}}{\alpha_{1}}\right)^{\beta_{1}} \frac{\Gamma\left(\frac{1+\beta_{1}}{\beta_{2}}\right)}{\Gamma\left(1 / \beta_{2}\right)}-\frac{\beta_{1}+\beta_{2}}{\beta_{1} \beta_{2}}
\end{aligned}
$$

where $\Gamma$ is the special Gamma function.

On the other hand, the magnitudes of the PolSAR spatial wavelet detail coefficients are modeled by WBL scale parameter $a>0$ and shape parameter $b>0$ so that if $X_{1}=$ $\left|\mathbf{W}_{j, n}\left[\mathcal{I}_{m}^{\mathcal{P}_{\epsilon}}\right]\right|$ and $X_{2}=\left|\mathbf{W}_{j, n}\left[\mathcal{I}_{\ell}^{\mathcal{P}_{\epsilon}}\right]\right|$ are the corresponding WBL variables, then the symmetric divergence of Eq. (9) has the following form [16]:

$$
\begin{aligned}
\mathcal{K}\left(X_{1}, X_{2}\right) & =\left(\frac{\lambda_{1}}{\lambda_{2}}\right)^{k_{2}} \Gamma\left(1+\frac{k_{2}}{k_{1}}\right)+\left(\frac{\lambda_{2}}{\lambda_{1}}\right)^{k_{1}} \Gamma\left(1+\frac{k_{1}}{k_{2}}\right) \\
& +\mathfrak{e}\left(\frac{k_{1}}{k_{2}}+\frac{k_{2}}{k_{1}}-2\right)-2+\left(k_{1}-k_{2}\right) \log \frac{\lambda_{1}}{\lambda_{2}}(14)
\end{aligned}
$$

where $\mathfrak{e}$ is the Euler-Mascheroni constant.

\section{APPLICATION}

This section is dedicated to experimental results on a multi-

\begin{tabular}{|c|c|c|}
\hline ROI label & Center point coordinates & Radius \\
\hline 01 & $03^{\circ} 58^{\prime} 44.55^{\prime \prime} \mathrm{N}-5143^{\prime} 30.57^{\prime \prime} \mathrm{W}$ & 10 \\
\hline 02 & $03^{\circ} 58^{\prime} 28.18^{\prime \prime} \mathrm{N}-5145^{\prime} 31.59^{\prime \prime} \mathrm{W}$ & 16 \\
\hline 03 & $03^{\circ} 53^{\prime} 04.31^{\prime \prime} \mathrm{N}-5145^{\prime} 09.66^{\prime \prime} \mathrm{W}$ & 09 \\
\hline 04 & $03^{\circ} 51^{\prime} 47.299^{\prime \prime} \mathrm{N}-5146^{\prime} 23.26$ 'W & 28 \\
\hline 05 & $03^{\circ} 50^{\prime} 59.68^{\prime \prime} \mathrm{N}-5145^{\prime} 09.50^{\prime \prime} \mathrm{W}$ & 11 \\
\hline 06 & $03^{\circ} 50 ' 53.64$ 'N - 5144'36.93'”W & 12 \\
\hline 07 & $03^{\circ} 50^{\prime} 45.144^{\prime \prime} \mathrm{N}-5146^{\prime} 12.23^{\prime \prime} \mathrm{W}$ & 06 \\
\hline 08 & $03^{\circ} 50 ' 44.44 " \mathrm{~N}-5145^{\prime} 11.21$ '” & 30 \\
\hline 09 & $03^{\circ} 50^{\prime} 04.76^{\prime \prime} \mathrm{N}-5145^{\prime} 44.71^{\prime \prime} \mathrm{W}$ & 10 \\
\hline 10 & $03^{\circ} 48^{\prime} 21.54^{\prime \prime} \mathrm{N}-5145^{\prime} 17.95^{\prime \prime} \mathrm{W}$ & 10 \\
\hline 11 & $03^{\circ} 45^{\prime} 46.39^{\prime \prime} \mathrm{N}-5145^{\prime} 26.45^{\prime \prime} \mathrm{W}$ & 16 \\
\hline 12 & $03^{\circ} 45^{\prime} 28.00^{\prime \prime} \mathrm{N}-5145^{\prime} 11.81^{\prime \prime} \mathrm{W}$ & 11 \\
\hline 13 & $03^{\circ} 38^{\prime} 55.02^{\prime \prime} \mathrm{N}-5135^{\prime} 08.28^{\prime \prime} \mathrm{W}$ & 99 \\
\hline
\end{tabular}
temporal Sentinel-1-A/B SAR time series acquired from 201512-05 to 2017-12-09 (87 dual-PolSAR GRD images). The latitudes of the test site range from $03^{\circ} 33^{\prime}$ to $04^{\circ} 26^{\prime}$ whereas
TABLE I

GEO-SPATIAL COORDINATES OF SOME AREAS WITH SUSPICIOUS GMWTV BEHAVIORS. THE ROI RADIUS IS GIVEN IN PIXELS, THE PIXEL SIZE BEING APPROXIMATELY $150 \mathrm{~m}^{2}$.

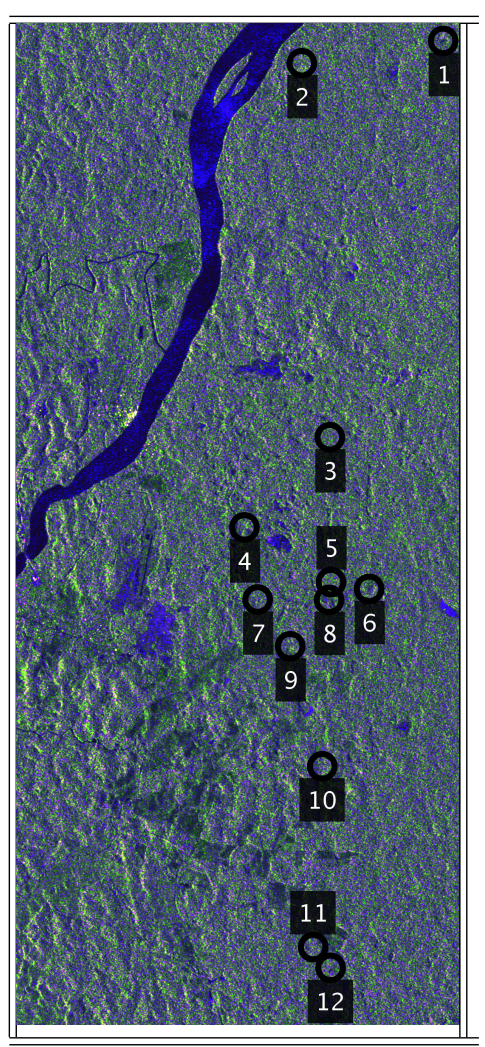

Fig. 2. Image sub-selection showing 12 ROIs among the 13 studied (see Table I for center pixel coordinates). The $13^{\text {th }}$ ROI is located far at southeast of ROI \#12. The display omits the latter because otherwise, the paper size leads to over-stretching all ROIs.

its longitudes range from $51^{\circ} 04^{\prime}$ to $51^{\circ} 54$ '. We follow the block diagram of Figure 1 for the analysis.

We select weights associated with the fused GMWTV of Eq. (6) from wavelet filter specifications. By imposing complementarity in the Fourier domain (high and band-pass filters), in addition with a significant attenuation of low frequencies (very slow variations in the time domain) we derive $\alpha_{2}=1 / 2$ and $\alpha_{1}=\alpha_{3}=1 / 4$.

GMWTV measures are then computed over pixel time series of the test site and the derived map highlights several types 


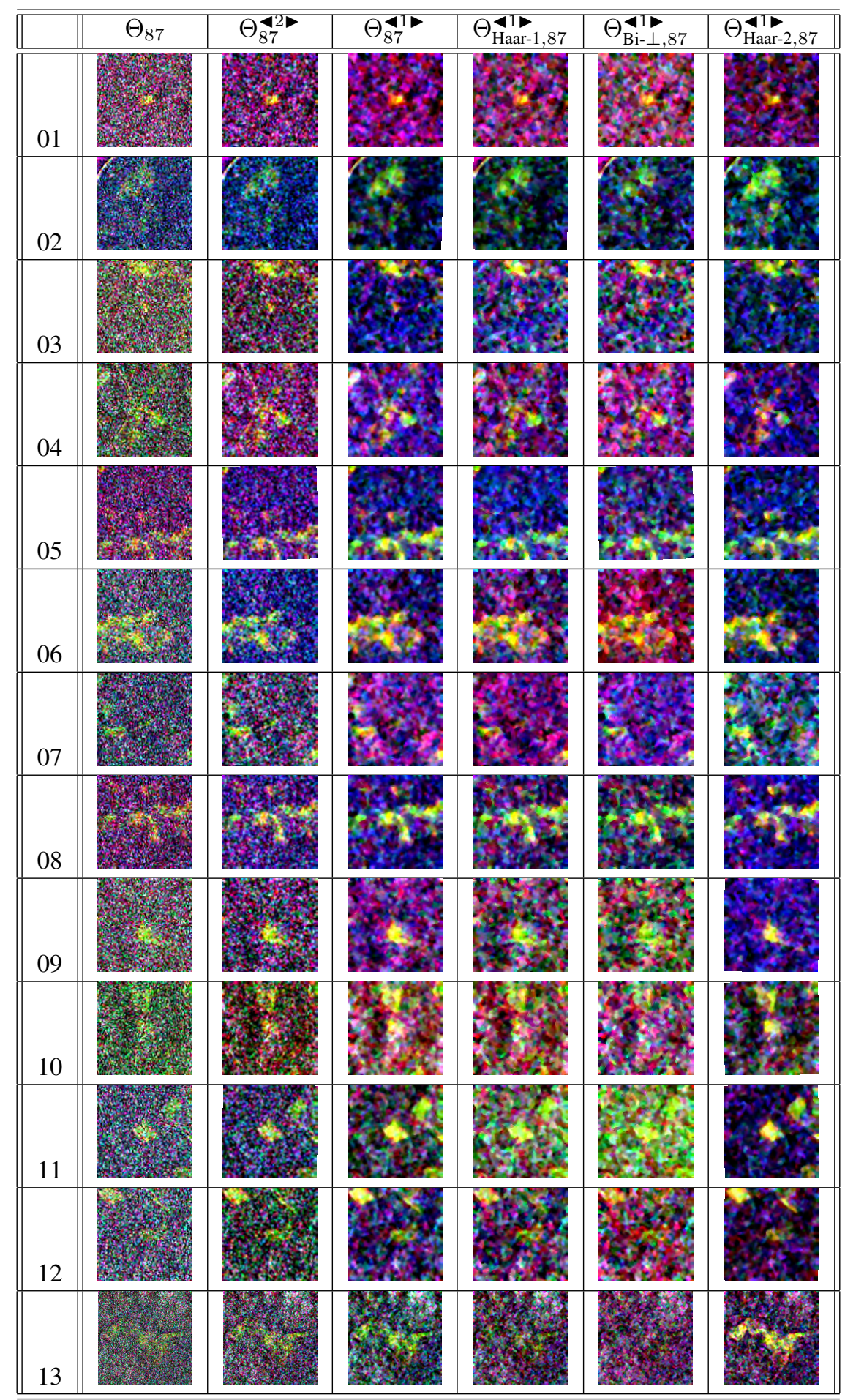

Fig. 3. $128 \times 128$ sub-selections of WTVs $\Theta_{\bullet}, 87$ and GMWTV $\Theta_{87}$ around the areas with abnormal behaviors presented in Table I.

of dynamics. The large size of the site does not allow fine displays on a limited length paper. Thus, we focus hereafter on 13 specific areas leading to large GMWTV: the coordinates of the centers for these 13 areas are given in Table I. Twelve among these areas are located in a tight neighborhood, close to the Oyapock river and are displayed in Figure 2.

GMWTV of the 13 areas described in Table I are given in
Figure 3. As it can be seen on this figure, the central pixels associated with the 13 areas have abnormal behaviors (bright colors). We also provide the size of the region surrounding any suspicious pixel behavior as a circular Region-Of-Interest (ROI) with radii specified in Table I.

Figure 3 shows that GMWTV $\Theta_{87}$ is noisy, as expected. Its $\ell^{2}$ regularized version $\Theta_{87}^{\mathbf{4}}$ is more informative, but 


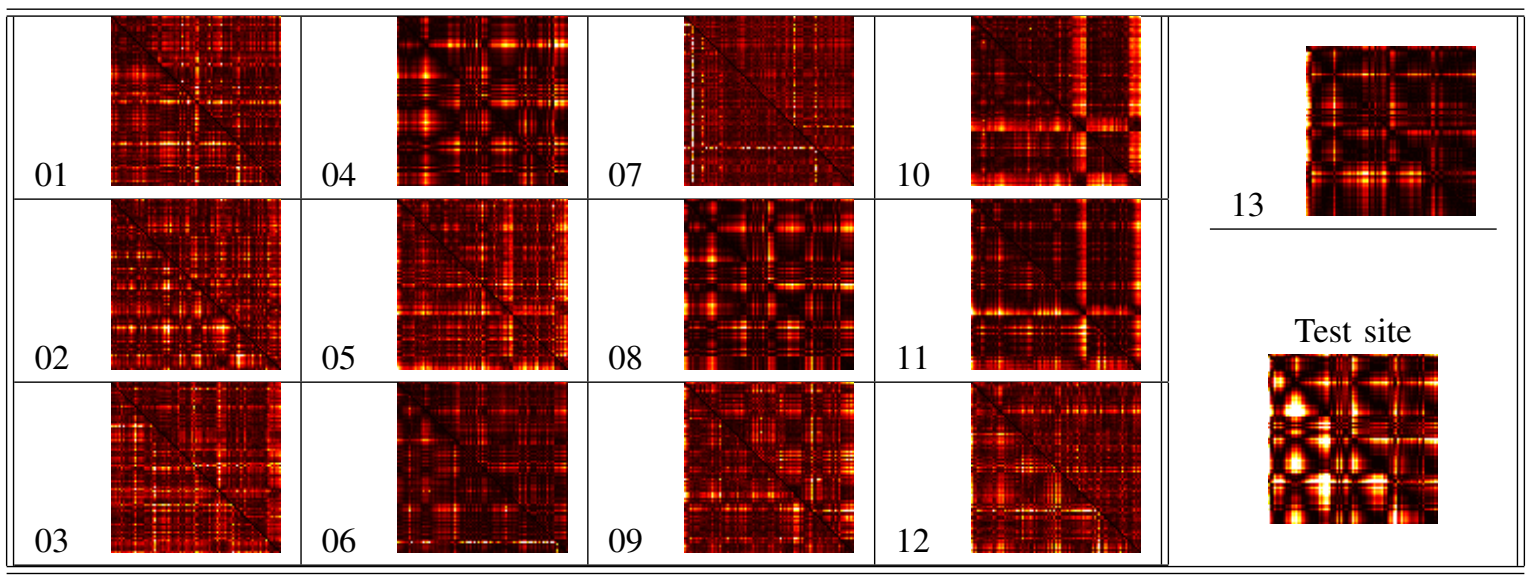

Fig. 4. MDDMs $\mathcal{K}$ for areas with suspicious behaviors given by Table $\mathrm{I}$ and for the test site.

less homogeneous than the recursive $\ell^{1}$ based regularization (compare columns associated with $\Theta_{87}^{41 \downarrow}$ and $\Theta_{87}^{42}$ ). In addition, GMWTV $\Theta_{87}^{1}$ (multi-wavelets) is more discriminant than any single wavelet GWTV: compare column associated with $\Theta_{87}^{41}$ to the columns associated respectively to Haar-1 $\left(\Theta_{\text {Haar- }-1,87}^{11}\right), \mathrm{Bi}-\perp\left(\Theta_{\text {Bi- }-1,87}^{1-}\right)$ and Haar-2 $\left(\Theta_{\text {Haar- } 2,87}^{1-1}\right)$ GWTVs.

Finally, asymmetric (VH, VV) MDDMs are derived for the 13 ROIs and displayed in Figure 4. The interpretation of these MDDMs according to color variations in rows, columns and diagonals let us derive the following conclusions:

- ROIs associated with a single change point are:

- Label 01 (abrupt change with seasonal variability);

- Label 02 (progressive changes with almost no visible seasonal variability).

- ROIs associated with multiple change points are:

- Labels 04, 06, 08, 09, 12, 13 (with visible seasonal variability);

- Labels 03, 05, 07, 10, 11 (no visible seasonality).

The MDDM of the whole test site is also given in Figure 4 for the sake of comparison: at this scale (more than $10^{8}$ pixels), this MDDM is dominated by seasonal changes mainly. This MDDM shows for instance that $\mathrm{VH}$ is more sensitive to seasonal variability than VV.

\section{CONCLUSION}

We have proposed SAR ITS analysis from a two stage information fusion. The first stage is a fusion of geometric wavelet operators in order to spot abnormal temporal scattering behaviors (high wavelet total variations that may issue from some abrupt change or cumulative seasonal effects). The second stage is a fusion of wavelet spatial multiscale divergences for capturing texture variations over time. The asymmetric MDDM proposed for the latter stage keeps available in a single matrix, both VV and VH PolSAR variabilities. It follows that certain changes/evolution are more visible on either $\mathrm{VV}$ or $\mathrm{VH}$ whereas others concern both $(\mathrm{VV}, \mathrm{VH})$ channels.

\section{REFERENCES}

[1] A. Garzelli and C. Zoppetti, "Optimizing sar change detection based on log-ratio features," in 2017 9th International Workshop on the Analysis of Multitemporal Remote Sensing Images (MultiTemp), June 2017.
[2] M. N. Sumaiya and R. S. S. Kumari, "Logarithmic mean-based thresholding for sar image change detection," IEEE Geoscience and Remote Sensing Letters, vol. 13, no. 11, pp. 1726-1728, Nov 2016.

[3] O. Antropov, Y. Rauste, F. M. Seifert, and T. Hme, "Selective logging of tropical forests observed using 1- and c-band sar satellite data," in 2015 IEEE International Geoscience and Remote Sensing Symposium (IGARSS), July 2015, pp. 3870-3873.

[4] C. Marin, F. Bovolo, and L. Bruzzone, "Building change detection in multitemporal very high resolution SAR images," IEEE Trans. Geosci. Remote Sens., vol. 53, no. 5, pp. 2664-2682, 2015.

[5] M. T. Pham, G. Mercier, and J. Michel, "Change detection between sar images using a pointwise approach and graph theory," IEEE Transactions on Geoscience and Remote Sensing, vol. 54, no. 4, pp. 2020-2032, April 2016.

[6] Y. Wang, L. Du, and H. Dai, "Unsupervised sar image change detection based on sift keypoints and region information," IEEE Geoscience and Remote Sensing Letters, vol. 13, no. 7, pp. 931-935, July 2016.

[7] W. Ren, J. Song, J. Zeng, and X. Zhang, "A novel asymmetrical probability density function for modeling log-ratio sar images," IEEE Geoscience and Remote Sensing Letters, vol. 13, no. 3, pp. 369-373, March 2016.

[8] A. Galletti and A. Maratea, "Numerical stability analysis of the centered log-ratio transformation," in 2016 12th International Conference on Signal-Image Technology Internet-Based Systems (SITIS), Nov 2016, pp. 713-716.

[9] A. M. Atto, E. Trouvé, J. M. Nicolas, and T. T. Lê, "Wavelet operators and multiplicative observation models - application to sar image timeseries analysis," IEEE Transactions on Geoscience and Remote Sensing, vol. 54, 2016.

[10] H. Mitchell, Image Fusion: Theories, Techniques and Applications. Springer-Verlag Berlin Heidelberg, 2010.

[11] K. Amolins, Y. Zhang, and P. Dare, "Wavelet based image fusion techniques an introduction, review and comparison," ISPRS Journal of Photogrammetry and Remote Sensing, vol. 62, no. 4, 2007.

[12] G. Pajares and J. M. de la Cruz, "A wavelet-based image fusion tutorial," Pattern Recognition, vol. 37, no. 9, pp. 1855 - 1872, 2004.

[13] A. M. Atto and G. Mercier, "High order structural image decomposition by using non-linear and non-convex regularizing objectives," Computer Vision and Image Understanding, vol. 138, no. Supplement C, pp. 38 50, 2015.

[14] A. M. Atto, E. Trouvé, Y. Berthoumieu, and G. Mercier, "Multidate divergence matrices for the analysis of sar image time series," IEEE Transactions on Geoscience and Remote Sensing, vol. 51, no. 4, April 2013.

[15] M. N. Do and M. Vetterli, "Wavelet-based texture retrieval using generalized gaussian density and kullback-leibler distance," IEEE Transactions on Image Processing, vol. 11, no. 2, pp. 146 - 158, Feb. 2002.

[16] R. Kwitt and A. Uhl, "Image similarity measurement by kullback-leibler divergences between complex wavelet subband statistics for texture retrieval," IEEE International Conference on Image Processing, ICIP, San Diego, California, USA, 12 - 15 October, pp. 933 - 936, 2008. 